\title{
Retrospective study of effect of post-operative radiotherapy on control of cancer urinary bladder stage two and three
}

\author{
Ali Mohamed Ali, Ahmed M. Ameen, Shimaa Roshdy Abdelaal \\ Clinical Oncology Department, Faculty of Medicine, Sohag University \\ Sohag Medical Journal Vol.21.3october 2017
}

\begin{abstract}
:
Aim of the work: evaluate benefit of post-operative radiotherapy in stage two and three bladder cancer after radical cystectomy as regard LRC, OS, DFS, and MFS and evaluate toxicity profile.

Patients and Method: A retrospective study of sixty two patients with pathologically proven bladder cancer who presented to the clinical oncology department, sohag University hospital from januray 2010 to December 2014. All of them underwent RC or anterior pelvic exenteration followed by post-operative radiotherapy after surgery for 5000 cGy in 25 fractions, over 5 weeks using 2D technique. This study was conducted by hand search in the files and radiotherapy sheet of these patients.

Results: Sixty two eligible bladder cancer patients were included in our study; it includes 39 males $(63 \%)$ and 23 females $(37 \%)$, with a male to female ratio of $1.7: 1$. The mean age was 58.15 years. Transitional cell carcinoma representing $(61 \%)$ of cases while SCC representing (39\%) of cases

Forty eight patients of our study developed acute and chronic toxicities (77\%), Out of 62 eligible bladder cancer patients, 2 patients $(3 \%)$ developed local recurrence, while 8 patients (13\%) developed distant metastasis, The 2, 3 and $5 \mathrm{yr}$ OS of the whole cohort of patients are $66 \%, 64 \%$ and $53.5 \%$ respectively, The 2, 3 and 5yr MFS of whole cohort of patients was $90 \%, 84 \%$ are $80.5 \%$ respectively, The 2, 3 and 5yr LRC of whole cohort of patients was $95 \%, 95 \%$ and $95 \%$ respectively.

Conclusion: In our retrospective study, post-operative radiotherapy showed good OS, DFS, LRCand MFS rates that were in agreement with some previous studies. Due to the small number of patients, the relatively short follow up periods and the statistical bias linked to retrospective design of clinical studies, further prospective studies with longer follow up periods are warranted to better evaluate such an effect better with using new radiotherapy modalities such as CRT and IMRT.
\end{abstract}

\section{Introduction:}

Bladder cancer is the ninth most common cancer worldwide and is more common in developing countries (1).In Egypt, bladder cancer incidence is much higher than reported in western countries, bladder cancer contributed $6.94 \%$ of all cancer in Egypt according to results of the National PopulationBased Registry Program of Egypt 2008-
2011, and males are more affected than females (2).

There are different histological subtypes of bladder cancer, For decades , SCC was considered the most common pathological subtype of cancer bladder in Egypt but this has changed greatly over the years, according to the NCI study in 2007, TCC predominated over $\operatorname{SCC}(3)$. 
Radical cystectomy (RC) and pelvic lymphdenectomy with or without neoadjuvant chemotherapy still remains the gold standard treatment for muscleinvasive bladder cancer (4).Loco regional recurrence following radical cystectomy for patients with muscle invasive UBC is common, its accounts for approximately $75 \%$ of the failures of this treatment (5).A meta-analysis of randomized controlled trials with or without platinum-based chemotherapy following local therapy (usually RC) showed that $25.6 \%$ of patients with chemotherapy had locoregional recurrence as a first event with or without synchronous distant metastasis (6).Local disease control is a clinically relevant challenge in the management of muscle invasive bladder carcinoma.In an attempt to increase locoregional control, the use of postoperative radiotherapy (PORT) was explored decades ago and demonstrated robust local control, Postoperative radiotherapy has the advantage of dealing with microscopic cells that are easier to sterilize (7).The radiation-induced late complications are the main limiting factor for the use of PORT after RC worldwide; however, the risk for radiation-induced complications can be minimized by novel radiotherapy techniques CRT or IMRT (8).

\section{Aim of the work:}

1. Evaluate benefits of post-operative radiotherapy in stage II \& III bladder cancer after radical cystectomy as regard LRC, OS, DFS \& DFFS.

2. Evaluate toxicity profile .

\section{Materials and Methods:}

Our study included 62 patients with pathologically proven bladder cancer who presented to the Oncology department, Sohag University Hospital, from January 2010 till December 2014. Informed consent was taken from all cases whenever possible.

$\checkmark$ Exclusion criteria:

Patients with end organ failure, patients with stage one or metastatic disease, patients with histological types other than (TCC, SCC, adenocarcinoma).

$\checkmark$ Methods

All the patients were subjected to radical cystectomy or anterior pelvic exenteration with urinary diversion and referred to our department for postoperative radiotherapy. The patients underwent clinical examination, routine labs, CT chest, abdomen \& pelvis. The patients were staged according to the American joint committee of cancer clinical staging system 2010. After being certain of no metastasis, the patients underwent simulation using our 2-D simulator machine to define volume to be treated. The patients were treated by 3 fields, one anterior and 2 lateral wedged fields aiming at delivering $50 \mathrm{GY} / 25$ fractions $/ 5$ weeks to isocenter by our 6 MV linear accelerator. The volume to be treated for all patients was as follow :

- Upper border: between lumbar vertebra five and the first sacral vertebra .

- Lower border: at the inferior border of obturator foramen.

- Lateral border: $1.5 \mathrm{~cm}$ outside the bony pelvic brim.

- The anterior border of the lateral field lies just in front of the symphysis pubis.

- The posterior border at the junction of anterior one third and posterior two thirds of the rectal circumference or the junction of the first and second sacral 
Follow up was done weekly during radiotherapy as regard evaluation of toxicity.The follow up visits after radiotherapy made every 3 months ,during which patient underwent clinical examination ,routine labs, radiological examination when indicated .

Assessment of outcomes of treatment was done by means of the following end points :

- Overall survival (OS): the period started from the date of diagnosis until patient death or time of last follow up.

- Disease free survival (DFS): the period started from the date of cystectomy until the first appearance of relapse (local, nodal or systemic) or the last date of follow up.

- Local control period: is the time started from the date of cystectomy until appearance of loco regional recurrence, or time of last follow up in those who did not develop local recurrence.

- Metastasis free survival (MFS): the period from cystectomy until first appearance of dissemination or time of last follow up in those who did not develop distant metastasis.

\section{Statistical analysis:}

Data was analyzed using STATA intercooled version 12.1. Quantitative data was represented as mean, standard deviation, median and range. Qualitative data was presented as number and percentage. Survival analysis was done using Kaplan-Meier method and comparison between two survival curves was done using log-rank test. Graphs were produced by using Excel or STATA program. $\mathrm{P}$ value was considered significant if it was less than 0.05 .

\section{Results:}

Sixty two patients who were fulfilling our eligibility criteria were included in our study, the follow up period ranged between 30 to 60 months with median at 36 months. The patient's age range between (30years-75years) with mean age 58.15years.

Follow up of the patients was done in Sohag University Hospital, Health Insurance, Sohag Cancer Institute. Some patients couldn't be assessed except by telephone.

Sixty two eligible bladder cancer patients were included in our study; it includes 39 males $(63 \%)$ and 23 females $(37 \%)$, with a male to female ratio of $1.7: 1$. The mean age was 58.15 years. Twenty patients were smokers $(32 \%), 3$ patients had history of bilharziasis $(5 \%), 9$ patients were hypertensive $(15 \%)$ and 6 patients were diabetic $(10 \%)$ as shown in table (1).

Transitional cell carcinoma representing $(61 \%)$ of cases while SCC representing (39\%) of cases. High grade tumors (grade 3) representing 58\% while low grade tumors representing $42 \%$. The commonest pathological stage was pT3b which was reported in $47 \%$ of patients followed by pT2b counted in $23 \%$ of patients as shown in table (2) Surgery in the form of radical cystectomy was performed for 50 patients constituting $(81 \%)$ of all patients, Anterior pelvic exenteration was performed for 12 patients constituting (19\%) of all patients. All patients received post-operative radiotherapy 50 GY in 25 fractions in 5 weeks withor without concurrent weekly chemotherapy. Thirty two $(52 \%)$ of the patients received concurrent chemotherapy, 8 of them $(25 \%)$ received carboplatin and $24(75 \%)$ received cisplatin. Thirty five of the cases $(56 \%)$ received their radiotherapy course in $\leq 40$ days as shown in table (3). 
Forty eight patients of our study developed acute and chronic toxicities (77\%), 41 patients $(66 \%)$ developed acute intestinal toxicity, 3 patients $(5 \%)$ developed late intestinal toxicity, 15 patients $(24 \%)$ developed acute skin toxicity, 12 patients developed acute renal toxicity and 8 patients developed chronic renal toxicity as shown in table (4). Out of 62 eligible bladder cancer patients, 2 patients $(3 \%)$ developed local recurrence, while 8 patients $(13 \%)$ developed distant metastasis, 4 of them (6\% of total sample) developed bone metastasis, 3(5\% of total sample) developed lung metastasis and only one ( $2 \%$ of total sample) developed liver metastasis as shown in table (5).

The 2,3 and 5yr OS of the whole cohort of patients are $66 \%, 64 \%$ and53.5\% respectively, on analyzing the impact of different clinico_ pathological factors as shown in table(6) we found that, age , biharzial infestion and pathological type significantly affected OS with P-value at $0.001,0.01$ and 0.04 respectively .

The 2, 3 and 5yr DFS of whole cohort of patients are $86 \%, 83 \%$ and79\% respectively, on analyzing the impact of different clinico_ pathological factors as shown in table(7), pathological type found significantly affected DFS(P-value 0.04 ).

The 2, 3 and 5yr LRC of whole cohort of patients was 95\%,95\% and 95\% respectively, on analyzing impact of different clinico_ pathological factors as shown in table (9), no factor found significantly affected LRC.

The 2, 3 and 5yr MFS of whole cohort of patients was $90 \%, 84 \%$ are $80.5 \%$ respectively, on analyzing impact of different clinico_ pathological factors as shown in table(8), no factor found significantly affected MFS.

Table (1): Patient's characteristics

\begin{tabular}{|l|c|c|}
\hline Characteristics & Number & Percentage (\%) \\
\hline Age & & \\
$\leq 65$ years & 43 & 69.35 \\
$>65$ years & 19 & 30.65 \\
\hline Gender & 23 & \\
Females & 39 & 37.10 \\
Males & & 62.90 \\
\hline Smoking & 21 & 33.87 \\
No & 20 & 32.26 \\
Yes & 21 & 33.87 \\
Unknown & & \\
\hline Bilharziasis & 32 & 51.61 \\
No & 3 & 4.84 \\
Yes & 27 & 43.55 \\
Unknown & & \\
\hline Hypertension & 36 & 58.06 \\
No & 9 & 14.52 \\
Yes & 17 & 27.42 \\
Unknown & & \\
\hline DM & 42 & 67.74 \\
No & 6 & 9.68 \\
Yes & 14 & 22.58 \\
\hline Unknown & & \\
\hline
\end{tabular}


SOHAG MEDICAL JOURNAL

Vol. 21 No.3 october 2017
Retrospective study of effect of post-operative radiotherapy

ShimaaRoshdyAbdelaal

Table (2): disease characteristics of studied populations

\begin{tabular}{|l|c|c|}
\hline Characteristics & Number & Percentage (\%) \\
\hline Surgical pathological type & 24 & 38.71 \\
SCC & 38 & 61.29 \\
TCC & 5 & 8.06 \\
\hline Surgical pathological grade & 21 & 33.87 \\
Grade 1 & 36 & 58.06 \\
Grade 2 & & 22.58 \\
Grade 3 & 14 & 14.52 \\
\hline T stage & 9 & 46.77 \\
T2b & 29 & 16.13 \\
T3a & 10 & \\
T3b & & \\
T4a & & \\
\hline
\end{tabular}

Table (3): treatment charcterestics

\begin{tabular}{|c|c|c|}
\hline Treatment given & Number & Percentage (\%) \\
\hline $\begin{array}{l}\text { Surgery } \\
\text { Anterior pelvic exenteration } \\
\text { Radical cystectomy }\end{array}$ & $\begin{array}{l}12 \\
50\end{array}$ & $\begin{array}{l}19.35 \\
80.65\end{array}$ \\
\hline $\begin{array}{l}\text { Diversion type } \\
\text { Neo bladder } \\
\text { Uretero colic } \\
\text { Uretro cutaneous }\end{array}$ & $\begin{array}{l}19 \\
11 \\
32\end{array}$ & $\begin{array}{l}30.64 \\
17.75 \\
51.61\end{array}$ \\
\hline $\begin{array}{l}\text { Post-operative radiotherapy } 50 \text { CGY /25 } \\
\text { fraction } / 5 \text { weeks }\end{array}$ & 62 & $100 \%$ \\
\hline $\begin{array}{l}\text { Concurrent chemotherapy } \\
\text { No } \\
\text { yes }\end{array}$ & $\begin{array}{l}30 \\
32\end{array}$ & $\begin{array}{l}48.39 \\
51.61\end{array}$ \\
\hline $\begin{array}{l}\text { Type of used chemotherapy } \\
\text { Weekly Carboplatin } \\
\text { Weekly cisplatin }\end{array}$ & $\begin{array}{c}8 \\
24\end{array}$ & $\begin{array}{l}25 \\
75\end{array}$ \\
\hline $\begin{array}{l}\text { Duration of radiotherapy/day } \\
\leq 40 \\
>40\end{array}$ & $\begin{array}{l}35 \\
27\end{array}$ & $\begin{array}{l}56.45 \\
43.55\end{array}$ \\
\hline
\end{tabular}

Table (4): Toxicity in studied populations

\begin{tabular}{|l|c|c|}
\hline Characteristics & Number & Percentage (\%) \\
\hline Toxicity & 14 & 22.58 \\
No & 48 & 77.41 \\
Yes & 21 & 33.87 \\
\hline Acute intestinal toxicity & 41 & 66.13 \\
No & & \\
Yes & 3 & 4.83 \\
\hline Chronic intestinal toxicity & 59 & 95.16 \\
Yes & & \\
No & 47 & 75.81 \\
& 15 & 24.19 \\
\hline Acute skin toxicity & 62 & 100 \\
No & & \\
Yes & 50 & 80.65 \\
\hline Chronic skin toxicity & 12 & 12.90 \\
No & & 87.1 \\
\hline Acute urinary toxicity & 54 & 12.9 \\
No & 8 & \\
Yes & & \\
\hline Chronic urinary toxicity & & \\
No & & \\
Yes & & \\
\hline
\end{tabular}


Table (5): local recurrence and distant metastasis in studied population

\begin{tabular}{|l|c|c|}
\hline Characteristics & Number & Percentage (\%) \\
\hline Local recurrence & 60 & 96.77 \\
No & 2 & 3.23 \\
Yes & 54 & 87.10 \\
\hline Distant metastasis & 4 & 6.45 \\
No & 1 & 1.61 \\
Bone & 3 & 4.84 \\
Liver & & \\
Lung & 36 & 58.06 \\
\hline Death & 26 & 41.94 \\
No & & \\
Yes & & \\
\hline
\end{tabular}

Table (6): Overall survival of studied cases and its relation to different factors

\begin{tabular}{|c|c|c|c|c|c|}
\hline Factors & Number & $\begin{array}{c}\text { Cum survival at } \\
2 \text { yrs } \%\end{array}$ & $\begin{array}{c}\text { Cum survival at } \\
3 \text { yrs } \%\end{array}$ & $\begin{array}{c}\text { Cum survival at } \\
5 \text { yrs } \%\end{array}$ & P-value \\
\hline Whole group & 62 & 66.09 & 64.25 & 53.41 & \\
\hline $\begin{array}{l}\text { Age } \\
\leq 65 \text { years } \\
>65 \text { years }\end{array}$ & $\begin{array}{l}43 \\
19\end{array}$ & $\begin{array}{l}76.74 \\
42.11\end{array}$ & $\begin{array}{l}74.19 \\
42.11\end{array}$ & $\begin{array}{l}66.53 \\
22.46\end{array}$ & 0.001 \\
\hline $\begin{array}{l}\text { Gender } \\
\text { Females } \\
\text { Males } \\
\end{array}$ & $\begin{array}{l}23 \\
39 \\
\end{array}$ & $\begin{array}{l}65.22 \\
66.57 \\
\end{array}$ & $\begin{array}{l}65.22 \\
63.67 \\
\end{array}$ & $\begin{array}{l}53.51 \\
52.90 \\
\end{array}$ & 0.93 \\
\hline $\begin{array}{l}\text { Smoking } \\
\text { No } \\
\text { Yes } \\
\text { Unknown }\end{array}$ & $\begin{array}{l}21 \\
20 \\
21\end{array}$ & $\begin{array}{l}47.62 \\
70.00 \\
80.95\end{array}$ & $\begin{array}{l}47.62 \\
64.62 \\
80.95\end{array}$ & $\begin{array}{l}31.75 \\
57.44 \\
67.25\end{array}$ & 0.06 \\
\hline $\begin{array}{l}\text { Bilharziasis } \\
\text { No } \\
\text { Yes } \\
\text { Unknown }\end{array}$ & $\begin{array}{c}32 \\
3 \\
27\end{array}$ & $\begin{array}{l}50.00 \\
66.67 \\
85.19\end{array}$ & $\begin{array}{l}50.00 \\
33.33 \\
85.19\end{array}$ & $\begin{array}{l}34.29 \\
33.33 \\
74.71\end{array}$ & 0.01 \\
\hline $\begin{array}{l}\text { Surgical pathological type } \\
\text { SCC } \\
\text { TCC }\end{array}$ & $\begin{array}{l}24 \\
38 \\
\end{array}$ & $\begin{array}{l}53.85 \\
73.68 \\
\end{array}$ & $\begin{array}{l}48.95 \\
73.68\end{array}$ & $\begin{array}{l}37.30 \\
63.77\end{array}$ & 0.04 \\
\hline $\begin{array}{l}\text { Surgical pathological grade } \\
\text { Grade } 1 \\
\text { Grade } 2 \\
\text { Grade } 3\end{array}$ & $\begin{array}{c}5 \\
21 \\
36\end{array}$ & $\begin{array}{c}100 \\
57.14 \\
66.67 \\
\end{array}$ & $\begin{array}{c}100 \\
57.14 \\
63.64 \\
\end{array}$ & $\begin{array}{c}100 \\
50.79 \\
47.25\end{array}$ & 0.23 \\
\hline $\begin{array}{l}\text { T stage } \\
\text { T2b } \\
\text { T3a } \\
\text { T3b } \\
\text { T4a }\end{array}$ & $\begin{array}{c}14 \\
9 \\
29 \\
10\end{array}$ & $\begin{array}{l}57.14 \\
88.89 \\
55.17 \\
90.00\end{array}$ & $\begin{array}{l}57.14 \\
76.19 \\
55.17 \\
90.00\end{array}$ & $\begin{array}{l}57.14 \\
76.19 \\
45.14 \\
56.52\end{array}$ & 0.34 \\
\hline $\begin{array}{l}\text { Duration of radiotherapy/day } \\
\leq 40 \\
>40\end{array}$ & $\begin{array}{l}35 \\
27\end{array}$ & $\begin{array}{l}62.86 \\
70.18\end{array}$ & $\begin{array}{l}59.86 \\
70.18\end{array}$ & $\begin{array}{l}49.37 \\
60.15\end{array}$ & 0.45 \\
\hline $\begin{array}{l}\text { Concurrent chemo therapy } \\
\text { Yes } \\
\text { No }\end{array}$ & $\begin{array}{l}32 \\
30\end{array}$ & & & & 0.07 \\
\hline
\end{tabular}


SOHAG MEDICAL JOURNAL

Vol. 21 No.3 october 2017
Retrospective study of effect of post-operative radiotherapy

ShimaaRoshdyAbdelaal

Table (7): Diseasefree survival of studied cases and its relation to different factors

\begin{tabular}{|c|c|c|c|c|c|}
\hline Factors & Number & $\begin{array}{c}\text { Cum survival at } \\
2 \text { yrs } \%\end{array}$ & $\begin{array}{c}\text { Cum survival at } \\
3 \text { yrs } \%\end{array}$ & $\begin{array}{c}\text { Cum survival at } \\
5 \text { yrs } \%\end{array}$ & P-value \\
\hline $\begin{array}{l}\text { Age } \\
\leq 65 \text { years } \\
>65 \text { years }\end{array}$ & $\begin{array}{l}43 \\
19\end{array}$ & $\begin{array}{l}91.65 \\
68.63\end{array}$ & $\begin{array}{l}87.66 \\
68.63\end{array}$ & $\begin{array}{l}82.79 \\
68.63\end{array}$ & 0.12 \\
\hline $\begin{array}{l}\text { Gender } \\
\text { Females } \\
\text { Males }\end{array}$ & $\begin{array}{l}23 \\
39\end{array}$ & $\begin{array}{l}85.52 \\
86.27\end{array}$ & $\begin{array}{l}85.52 \\
80.88\end{array}$ & $\begin{array}{r}77.75 \\
80.88\end{array}$ & 0.78 \\
\hline $\begin{array}{l}\text { Bilharziasis } \\
\text { No } \\
\text { Yes } \\
\text { Unknown }\end{array}$ & $\begin{array}{c}32 \\
3 \\
27\end{array}$ & $\begin{array}{l}84.08 \\
50.00 \\
91.83\end{array}$ & $\begin{array}{l}74.73 \\
50.00 \\
91.83\end{array}$ & $\begin{array}{l}74.73 \\
50.00 \\
86.09\end{array}$ & 0.25 \\
\hline $\begin{array}{l}\text { T stage } \\
\text { T2b } \\
\text { T3a } \\
\text { T3b } \\
\text { T4a }\end{array}$ & $\begin{array}{c}14 \\
9 \\
29 \\
10\end{array}$ & $\begin{array}{l}77.78 \\
87.50 \\
87.67 \\
90.00\end{array}$ & $\begin{array}{l}62.22 \\
87.50 \\
87.67 \\
90.00\end{array}$ & $\begin{array}{l}62.22 \\
87.50 \\
87.67 \\
67.50\end{array}$ & 0.74 \\
\hline $\begin{array}{l}\text { Duration of } \mathrm{r} \\
\leq 40 \\
>40\end{array}$ & $\begin{array}{l}35 \\
27\end{array}$ & $\begin{array}{l}83.56 \\
89.72\end{array}$ & $\begin{array}{l}79.16 \\
89.72\end{array}$ & $\begin{array}{l}74.22 \\
89.72\end{array}$ & 0.23 \\
\hline
\end{tabular}

Table (8): distantmetastasis free survival of studied cases and its relation to different factors

\begin{tabular}{|c|c|c|c|c|c|}
\hline Factors & Number & $\begin{array}{c}\text { Cum survival at } \\
2 \text { yrs } \%\end{array}$ & $\begin{array}{c}\text { Cum survival at } \\
3 \text { yrs } \%\end{array}$ & $\begin{array}{c}\text { Cum survival at } \\
5 \text { yrs } \%\end{array}$ & P-value \\
\hline Whole group & 62 & 89.95 & 84.44 & 80.60 & \\
\hline $\begin{array}{l}\text { Age } \\
\leq 65 \text { years } \\
>65 \text { years }\end{array}$ & $\begin{array}{l}43 \\
19\end{array}$ & $\begin{array}{l}94.45 \\
76.69\end{array}$ & $\begin{array}{l}90.35 \\
65.73\end{array}$ & $\begin{array}{l}85.33 \\
65.73\end{array}$ & 0.08 \\
\hline $\begin{array}{l}\text { Gender } \\
\text { Females } \\
\text { Males }\end{array}$ & $\begin{array}{l}23 \\
39\end{array}$ & $\begin{array}{l}90.34 \\
89.51\end{array}$ & $\begin{array}{l}83.89 \\
83.92\end{array}$ & $\begin{array}{l}76.26 \\
83.92\end{array}$ & 0.61 \\
\hline $\begin{array}{l}\text { Smoking } \\
\text { No } \\
\text { Yes } \\
\text { Unknown }\end{array}$ & $\begin{array}{l}21 \\
20 \\
21\end{array}$ & $\begin{array}{l}88.89 \\
93.33 \\
89.16\end{array}$ & $\begin{array}{l}66.67 \\
93.33 \\
89.16\end{array}$ & $\begin{array}{l}66.67 \\
93.33 \\
81.73\end{array}$ & 0.20 \\
\hline $\begin{array}{l}\text { Bilharziasis } \\
\text { No } \\
\text { Yes } \\
\text { Unknown }\end{array}$ & $\begin{array}{c}32 \\
3 \\
27\end{array}$ & $\begin{array}{c}92.98 \\
0.50 \\
91.83\end{array}$ & $\begin{array}{l}77.48 \\
50.00 \\
91.83\end{array}$ & $\begin{array}{l}77.48 \\
50.00 \\
86.09\end{array}$ & 0.29 \\
\hline $\begin{array}{l}\text { Surgical pathological grade } \\
\text { Grade } 1 \\
\text { Grade } 2 \\
\text { Grade } 3 \\
\end{array}$ & $\begin{array}{c}5 \\
21 \\
36 \\
\end{array}$ & $\begin{array}{c}100 \\
82.54 \\
91.67 \\
\end{array}$ & $\begin{array}{c}100 \\
74.29 \\
87.08 \\
\end{array}$ & $\begin{array}{c}100 \\
74.29 \\
77.41 \\
\end{array}$ & 0.42 \\
\hline $\begin{array}{l}\text { T stage } \\
\text { T2b } \\
\text { T3a } \\
\text { T3b } \\
\text { T4a } \\
\end{array}$ & $\begin{array}{c}14 \\
9 \\
29 \\
10\end{array}$ & $\begin{array}{l}87.50 \\
87.50 \\
91.71 \\
90.00 \\
\end{array}$ & $\begin{array}{l}70.00 \\
87.50 \\
85.16 \\
90.00 \\
\end{array}$ & $\begin{array}{l}70.00 \\
87.50 \\
85.16 \\
67.50 \\
\end{array}$ & 0.93 \\
\hline $\begin{array}{l}\text { Duration of radiotherapy/day } \\
\leq 40 \\
>40\end{array}$ & $\begin{array}{l}35 \\
27\end{array}$ & $\begin{array}{l}86.84 \\
94.44\end{array}$ & $\begin{array}{l}78.53 \\
94.44\end{array}$ & $\begin{array}{l}73.62 \\
94.44\end{array}$ & 0.12 \\
\hline
\end{tabular}


Table (9) LRCof studied cases and its relation to different factors

\begin{tabular}{|c|c|c|c|c|c|}
\hline Factors & Number & $\begin{array}{c}\text { Cum survival at } \\
2 \text { yrs } \%\end{array}$ & $\begin{array}{c}\text { Cum survival at } \\
3 \text { yrs } \%\end{array}$ & $\begin{array}{c}\text { Cum survival at } \\
5 \text { yrs } \%\end{array}$ & $\mathrm{P}$-value \\
\hline $\begin{array}{l}\text { Age } \\
\leq 65 \text { years } \\
>65 \text { years }\end{array}$ & $\begin{array}{l}43 \\
19 \\
\end{array}$ & $\begin{array}{l}96.67 \\
90.00 \\
\end{array}$ & $\begin{array}{l}96.67 \\
90.00 \\
\end{array}$ & $\begin{array}{l}96.67 \\
90.00 \\
\end{array}$ & 0.32 \\
\hline $\begin{array}{l}\text { Gender } \\
\text { Females } \\
\text { Males } \\
\end{array}$ & $\begin{array}{l}23 \\
39 \\
\end{array}$ & $\begin{array}{l}94.44 \\
95.83 \\
\end{array}$ & $\begin{array}{l}94.44 \\
95.83 \\
\end{array}$ & $\begin{array}{l}94.44 \\
95.83 \\
\end{array}$ & 0.72 \\
\hline $\begin{array}{l}\text { Bilharziasis } \\
\text { No } \\
\text { Yes } \\
\text { Unknown }\end{array}$ & $\begin{array}{c}32 \\
3 \\
27 \\
\end{array}$ & $\begin{array}{c}89.67 \\
100 \\
100 \\
\end{array}$ & $\begin{array}{c}89.67 \\
100 \\
100 \\
\end{array}$ & $\begin{array}{c}89.67 \\
100 \\
\end{array}$ & 0.30 \\
\hline $\begin{array}{l}\text { Surgical pathological grade } \\
\text { Grade } 1 \\
\text { Grade } 2 \\
\text { Grade } 3\end{array}$ & $\begin{array}{c}5 \\
21 \\
36\end{array}$ & $\begin{array}{c}100 \\
92.31 \\
96.30\end{array}$ & $\begin{array}{c}100 \\
92.31 \\
96.30\end{array}$ & $\begin{array}{c}100 \\
92.31 \\
96.30 \\
\end{array}$ & 0.81 \\
\hline $\begin{array}{l}\text { T stage } \\
\text { T2b } \\
\text { T3a } \\
\text { T3b } \\
\text { T4a } \\
\end{array}$ & $\begin{array}{c}14 \\
9 \\
29 \\
10\end{array}$ & $\begin{array}{c}85.71 \\
100 \\
95.65 \\
100 \\
\end{array}$ & $\begin{array}{c}85.71 \\
100 \\
95.65 \\
100 \\
\end{array}$ & $\begin{array}{c}85.71 \\
100 \\
95.65 \\
100 \\
\end{array}$ & 0.62 \\
\hline $\begin{array}{l}\text { Duration of radiotherapy/das } \\
\leq 40 \\
>40\end{array}$ & $\begin{array}{l}35 \\
27 \\
\end{array}$ & $\begin{array}{l}96.00 \\
94.74 \\
\end{array}$ & $\begin{array}{l}96.00 \\
94.74 \\
\end{array}$ & $\begin{array}{l}96.00 \\
94.74 \\
\end{array}$ & 0.96 \\
\hline
\end{tabular}

\section{Discussion:}

Bladder cancer is a common malignancy in Egypt; it is representing $6.94 \%$ of all cancers (2).

In this retrospective study, 62 patients with bladder cancer presented to the clinical oncology department in Sohag University Hospital, all underwent RC and received PORT. Several epidemiological and clinical factors were studied aswell as prognostic factors influencing local tumor control, distant disease failure in addition to survivalBladder cancer is typically diagnosed in older individuals; with a median age at time of diagnosis is 73 . It is more common in males than in females with a $3: 1$ ratio (3).

In our study $69.35 \%$ of patients were $\leq$ 65 years at time of diagnosis with mean age at 58.15 year. The male to female ratio is (1.7:1).Younger age has better 5ys OS that is $66.5 \%$ for patients $\leq 65 \mathrm{yr}$ versus $22.5 \%$ for patient $>65 \mathrm{yr}$ ( $\mathrm{p}$ - value 0.001 ), this may be due to comorbid disease association with older age as shown in table (6).

Transitional cell carcinoma was found more prevalent in our cohort of patients representing $61 \%$ versus squamous cell carcinoma that found in $39 \%$ of patients asseen in table (2), also association with biharzial infestation was seen in small percent of our patients (4\%).Such these findings also observed by other investigators such as Gouda I and colleagues who conducted a study on 9843 patients in NCI, Cairo Egypt and found that $65.8 \%$ of patients were TCC and association with bilharzial 
infestation was found in 55\% in contrast to $82 \%$ in older series. (3)

Postoperative radiotherapy led to marked reduction in the incidence of local recurrence and henceimproved the disease-free survival in bladder cancer patients (7). Many Egyptian series had been performed to assess postoperative radiotherapy and its influence on survival. Well-conducted retrospective randomized series reported that postoperative radiotherapy improved disease free survival concerning different stages, different grades and lymph nodal status (12).The beneficial effect of PORT was proved in a large prospective randomized trial at NCICairo, including 236 patients, for locally advanced bladder cancer patients. The 5 year LC rates were $87 \% \pm 4 \%$ and $93 \%$ $\pm 3 \%$ for hyperfractionated (HF) and conventional (CF) PORT respectively compared to $50 \% \pm 6 \%$ for the cystectomy alone group. This effect was consistent across all tumor types, grades and pathologic stages whether or not the lymph nodes were involved. The 5-year LC rates for pT3a, pT3b, and pT4a were $96 \%, 91 \%$, and $74 \%$ respectively (7).These results were confirmed in nonrandomized controlled Radiation Therapy Oncology Group (RTOG) trials, Cozzarini et al. (1999) and other Egyptian and non-Egyptian series for patients having TCC, SCC, and adenocarcinoma(12). Better DFS and LC rates with PORT were also reported by Reisinger et al. 1992) who found that, DFS rates ranged between $45 \%$ - 79\% while LC rates ranged between $79 \%$ $97 \%$ in patients received PORT(13).

In 2015, Nasr and colleagues conducted a study to reevaluate this protocol regarding its effect on prognosis and complications, the results were published in 2015 and showed high LC rates at $93 \%$ similar to results described previously by Zaghloul et al. 1992 . (12)Depending on these results, the routine work at NCI-Cairo was to give PORT for bladder cancer patients with $\mathrm{T}$-stage $\geq \mathrm{pT} 2 \mathrm{~b}$ or node positive cases(12).

In our study 3-years LRC is $95 \%$ for whole group, 3-years DFS is $83 \%$ for whole group and 3-years OS is $64 \%$ for whole group as shown in table $(6,7$ and 9).

Synchronous chemo radiotherapy may have advantages over radiotherapy alone, only one randomized trial has compared these two approaches in bladder cancer which reported that addition of chemotherapy to standarddose radiotherapy was associated with a relative reduction of $33 \%$ in the risk of loco regional recurrence with a reduction of almost 50\% in invasive recurrences compared with radiotherapy alone, and resulted in good long-term bladder function. (14).In our study concurrent weekly chemotherapy has no impact on survival (p-value 0.07 ) as shown in table (6).

With reference to period of overall treatment, prolongation of overall period of delivery of postoperative radiotherapy reduces the local control rate for bladder cancer (18).In Zaghloul,et al 2002, Those whose overall treatment periods were of 40 days or less had 2-year DFS of $77.6 \pm 8 \%$ compared to $46.8 \pm 16 \%$ for those received treatment in more than 40 days(17).But this is not reported in Safwat and Bond study that did not find a significant effect of unplanned treatment gap on the local control rate (19).

In our study, Those whose overall treatment periods were of 40 days or less had 2-yr LRC rate of $96 \%$ compared to $94.7 \%$ for those received treatment in 
more than 40 days with P-value (0.96) as shown in table (9). This was also going with Safwat and Bond study that showed that the duration of treatment had no significant impact on local control.

The incidence of distant metastasis was low in early Egyptian bladder cancer series ranged between $2-5.8 \%$ (21). While in the recent reports systemic metastasis had as much higher incidence at $23 \%$. (20). This change was probably due to the addition of adjuvant local measures either in the form of preoperative or postoperative radiotherapy that had led to better local control and subsequently a longer disease-free survival which allowed a greater number of distant metastasis to manifest and appear, The independent risk factors for distant metastasis and local control were: pelvic lymph node involvement $(\mathrm{p}=0.005)$, pathological stage $(p=0.004)$ and histo pathological grade $(\mathrm{p}=0.05)(7)$.

In the present study distant metastasis occurred in 8 patients $(13 \%)$. The $3-y r$ DFFS rate is $84.5 \%$ that means that the cumulative distant metastases rate at 3$\mathrm{yr}$ is $15.5 \%$, an incidence similar to that reported earlier by Zaghloul 1996 as shown in table (7). Four of these reported distant metastasis were in bone $(50 \%)$, three in the lung $(37.5 \%)$, and one in the liver $(12.5 \%)$ as shown in table (5).

Post-operative radiotherapy in bladder cancer remains unpopular owing to the fear of late intestinal complications. Warning results were published by Reisinger et al. (1992) as they reported $37 \%$ (15 out of 40 patients) rate of intestinal obstruction in patients who received PORT. Nine out of these 15 patients required surgery and three died (13).In contrast, Zaghloul et al. (1992) had 14 out of the 78 patients $(18 \%)$ treated with post-operative conformal radiotherapy (CRT) and four out of the 75 patients $(5 \%)$ treated with hypo fractionated (HF) PORT, experienced chronic enteritis of different grades. Only three (4\%) and four (5\%) out of the CRT and HF patients, respectively, progressed to fistula that necessitated surgery (7).Similar complications were experienced by adenocarcinoma patients treated with PORT in a nonrandomized study comprising 192 patients after RC and pelvic lymphadenectomy (19).The difference in late sequelae between the latter two studies and that of Reisinger et al. (1992) was probably due to the larger volume of radiation used in the Reisinger et al. (1992) study (upper border at fourth Lumbar vertebrae) or the biologically higher dose reaching intestine as patients were given 500 CGy single dose preoperatively followed by 4000- 4500 CGy CF postoperatively, which led to severe late bowel complications .Also, Nasr et al. (2015) study documented $\sim 71 \%$ (148 patients) suffered from small bowel reactions (diarrhea/abdominal colic) mostly of grade 1 and 2 intensity with $55 \%$ and $43 \%$ respectively. Bleeding per rectum was encountered in only 4 patients (3\%). Grade 4 early bowel reactions were not experienced in any of the 208 patients. As for the late toxicity, 24\% suffered late reactions; lower limb edema, scrotal swelling, ureteric stricture. Only 6 patients $(3 \%)$ suffered from intestinal obstruction, in this study the figure was much less than that seen in Reisenger et al. (1992) study and claiming the continuous benefit of PORT with low complications that were seen in Zaghloul et al. (2006) study (12).

In our study 48 out of 62 patients $(77.5 \%)$ developed toxicities, 41 patients $(66 \%)$ had shown acute intestinal 
toxicities (Increased frequency or change in quality of bowel habits / rectal discomfort), most of them grade 1 and 2 and did not need any surgical intervention, 15 patients (24\%)developed acute skin toxicities (erythema / epilation / dry desquamation), most of them grade 1 and 2 , only 3 patients (5\%) with chronic intestinal toxicities as shown in table (4).

\section{References}

1-Torre LA, Bray F, et al (2015): Global cancer statistics, 2012. CA Cancer J Clin; 65:87.

2-Amal S. Ibrahim, Hussein M. Khaled, et al (2014): Journal of Cancer Epidemiology Volume 2014 (2014), Article ID 437971, 18 pages.

3-Gouda, I., Mokhtar, N., Bilal, D., et al (2007);Bilharziasis and Bladder Cancer: Journal of the Egyptian National Cancer Institute, 19, 158-162.

4-Bolenz, C, Ho, R, Nuss, G.R, et al. (2010) ,BJU International, 106, 13241329.

5-Ghoneim MA, El-Mekresh MM, El-Baz MA, (1997): Radical cystectomy for carcinoma of bladder: Critical evaluation of the results of 1026 cases. J. Urol., 158: 393.

6-Abol-Enein, H., et al. (2005): Neoadjuvant Chemotherapy for Invasive Bladder Cancer. Cochrane Database of Systematic Reviews, Article ID: CD005246
7-Zaghloul MS, Awwad HK, (1992),Int J Radiat Oncol Biol Phys; 23: 511-7.

8-Madersbacher S, Schmidt J, Eberle JM, et al (2003): Long-term outcome of ileal conduit diversion. J Urol; 169: 985.

9-Reisinger SA, Mohiuddin M,et al (1992): Combined pre- and postoperative adjuvant radiation therapy for bladder cancer. Int J Radiat Oncol Biol Phys; 24: 463-8

10-Nicholas D. James, Syed A. Hussain,et al (2012) ,N Engl J Med 2012; 366:14771488 .

11-Balci, U., Ozer, K., Gorgel, S.N., Sefik, E., et al (2013): World Journal of Urology, 31, 1177-1182.

12-De-Neve W., Lybeert M.L., ,et al (1995): Radiotherapy for 72 T3 carcinoma of the bladder: the influence of over all treatment time. Radiather Oncol: 36: 183-188.

13-Safwat A. and Bond S,et al (1999): Unplanned treatment gaps in post-operative, Radiotherapy of carcinoma in the bilharzial bladder, the results of an audit. J. Egypt Nat. Cancer Inst., 11: 271-277.

14-Zaghloul, M.S., Nouh, A., Nazmy, Zaghloul, A.S., et al ( 2006) ,Urologic Oncology: 24, 13-20 .

15-Zaghloul M.S,et al (1996): Distant metastasis from bilharzial blad- der cancer.

Cancer 77: 743-749

16-El-Sebai I, et al (1983): Bladder cancer, CRC Press, Vol., II: 163-197. 